\title{
Characterization of Silicon Nanowire Embedded in a MEMS Diaphragm Structure Within Large Compressive Strain Range
}

\author{
Liang Lou, Woo-Tae Park, Member, IEEE, Songsong Zhang, Li Shiah Lim, Dim-Lee Kwong, Fellow, IEEE, and \\ Chengkuo Lee, Member, IEEE
}

\begin{abstract}
The characteristics of piezoresistive silicon nanowires (SiNWs) under compressive strain as large as $1.7 \%$ are reported. The SiNW is embedded in a multilayered diaphragm structure consisting of silicon nitride and silicon oxide. After leveraging the high fracture stress and intrinsic tensile stress of silicon nitride layer to produce a flat diaphragm, we can create large compressive strain to the SiNW without damaging the diaphragm. The relationship between $\mathrm{SiNW}$ resistance change and applied strain is measured and investigated with $2-\mu \mathrm{m}$ and $5-\mu \mathrm{m}$ SiNWs for both scientific and practical points of view. This approach demonstrates the validity to reveal the SiNW properties under large strain, and the exploration provides good reference for future SiNW-based MEMS sensor design.
\end{abstract}

Index Terms-Large compressive strain, multilayered diaphragm structure, silicon nanowire (SiNW).

\section{INTRODUCTION}

B Y LEVERAGING the advanced semiconductor process technology, NEMS-based biosensors using silicon nanowire (SiNW) have been reported as promising DNA and protein sensors [1], [2]. Due to the large piezoresistive effect of SiNWs [3]-[5], using SiNWs as the piezoresistive sensing element in the pressure-deformable diaphragm operated in the tensile strain region has been reported [6]. Major research efforts have been devoted to the characterization of SiNW mechanical properties based on miniaturized or microelectromechanical systems (MEMS)-based testing platform [7][9]. Generally, these characterization approaches are kinds of method using the bulky test platform to conduct the four-point

Manuscript received August 24, 2011; revised September 16, 2011; accepted September 18, 2011. Date of publication October 26, 2011; date of current version November 23, 2011. This work was supported in part by the National University of Singapore under Grant MOE 2009-T2-011 (R263000598112) and in part by SERC, Agency for Science, Technology and Research, under Grants 0921480070, 1021010022, 1021650084, and 1021520013. The review of this letter was arranged by Editor W. T. Ng.

L. Lou is with the Institute of Microelectronics, Agency for Science, Technology and Research, Singapore 138632, and also with the Department of Electrical and Computer Engineering, National University of Singapore, Singapore 117576.

W.-T. Park, L. S. Lim, and D.-L. Kwong are with the Institute of Microelectronics, Agency for Science, Technology and Research, Singapore 138632 (e-mail: parkwt@ime.a-star.edu.sg).

S. Zhang and C. Lee are with the Department of Electrical and Computer Engineering, National University of Singapore, Singapore 117576 (e-mail: elelc@nus.edu.sg).

Color versions of one or more of the figures in this letter are available online at http://ieeexplore.iee.org.

Digital Object Identifier 10.1109/LED.2011.2169931 bending for uniform stress [7] or utilizing specially designed MEMS-based test platforms [8], [9]. However, due to fracture of samples under test, the four-point-bending setup provides very limited tensile/compressive strain usually within $0.06 \%$ [3]. On the other hand, characterization of SiNWs under large range of tensile strain has been reported by using MEMS-based testing platform [8]-[10]. For example, Lugstein et al. reported the $\langle 111\rangle$ direction SiNWs with applied strain as large as 3.5\% and claimed that the SiNW resistivity drops as the SiNW is elongated exceeding $0.8 \%$ strain [8]. However, up-to-date only piezoresistive property of SiNWs of large tensile strain has been reported, while the measured data of compressive strain are usually limited to $0.06 \%$ [3]. Four-point-bending bulky test platform suffers the fracture issue under large compressive strain, and MEMS-device-based testing platform usually measures a SiNW by artificially assembling the SiNW to the MEMS device or with synthesized SiNW, in which such SiNWs are restricted in a small compressive strain range due to buckling issues. In practical applications, the SiNWs are usually embedded in the thin films to form a sensor structure which is used not only in tensile state but also in compressive state. Therefore, as such investigation of SiNW behavior within a wide range of compressive strain is indispensable. In the recent report by Kozlovskiy and Sharan, the SiNW under large compressive stress $(\sim 1 \mathrm{GPa})$ is computationally investigated [11]. Here, we report a MEMS diaphragm consisting of SiNW piezoresistors for pressure sensor applications characterized in the large compressive strain range. By leveraging the large fracture stress of silicon nitride, the measured resistance data versus compressive strain up to $1.7 \%$ are recorded for SiNWs in $\langle 110\rangle$ direction.

\section{DESIGN AND FABRICATION}

The schematic drawing of the pressure sensor is shown in Fig. 1(a), while the SEM photo and optical microscope (OM) photo of a microfabricated multilayered diaphragm with a diameter of $200 \mu \mathrm{m}$ are shown in Fig. 1(b) and (d), while the zoom-in image of SiNWs is shown in Fig. 1(c). By confining the SiNW in the surrounding oxide, large compressive strain could be applied on this flat diaphragm structure to the extent before the diaphragm reaches the fracture point without buckling issues.

The process flow to fabricate the diaphragm starts with a $\mathrm{Si}$ (100) wafer. After photolithography, the photoresist is trimmed 

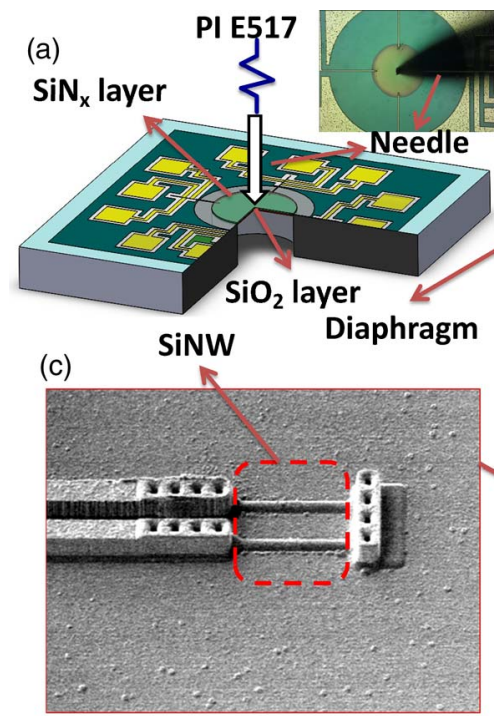

(b)

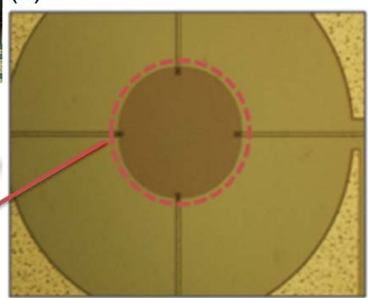

(d)

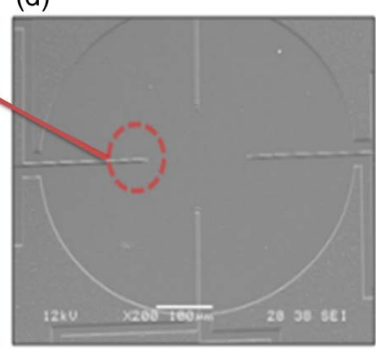

Fig. 1. (a) Schematic drawing of the pressure sensor with inset of $\mathrm{OM}$ photograph of needle tip. (b) OM photograph of the fabricated device. (c) SEM photograph of 5- $\mu \mathrm{m}$ SiNWs after metal deposition and contact via patterning. (d) SEM photograph of the fabricated device.

to decrease the critical dimension to around $100 \mathrm{~nm}$ for the following silicon etching. Thermal oxidation is conducted then to further shrink down the dimension of the SiNW. Diaphragms integrated with two kinds of SiNWs are made, while the cross section of both SiNWs is $90 \mathrm{~nm} \times 90 \mathrm{~nm}$ and the lengths of both SiNWs are $2 \mu \mathrm{m}$ and $5 \mu \mathrm{m}$, respectively. The piezoresistive characteristics of SiNWs are created by a p-type implantation process using $\mathrm{BF}_{2}+$ with a dosage of $1 \mathrm{E} 14 \mathrm{ion} / \mathrm{cm}^{2}$ and followed by annealing for activation. Next, an extra $\mathrm{SiO}_{2}$ layer of $4000 \AA$ is deposited for passivation; after via opening and metal patterning, 2.5- $\mu \mathrm{m}$ silicon nitride film is deposited to compensate the compressive stress in the $\mathrm{SiO}_{2}$ layer. Finally, DRIE is conducted to release the diaphragm structure.

\section{Modeling AND Characterization}

A white light interferometer (Vecco NT3300) system is used to record the surface profile of the microfabricated diaphragm as shown in Fig. 2(a). Fig. 2(b) shows the measured surface roughness across the membrane. These data give almost zero deflection, indicating that the SiNW is basically at a normal state without prestress. To investigate the diaphragm strain distribution, particularly at the SiNW area under a center point displacement load, finite-element analysis software ABAQUS is used in a nonlinear analysis mode. A three-layer structure model is built consisting of 1450- $\AA$ BOX layer, 4000- $\AA$ PECVD oxide, and 2.5- $\mu \mathrm{m}$ SiN layer as shown in Fig. 2(c) and (d). The SiNW is located between the two oxide layers at the diaphragm edge. The longitudinal strain of the SiNW is extracted and averaged from the elements at the membrane edge as the displacement load is given from 0 to $20 \mu \mathrm{m}$. The simulation results show that the strain of the diaphragm edge is in a fairly good linear relationship against the center displacement (Fig. 3 inset). (a)

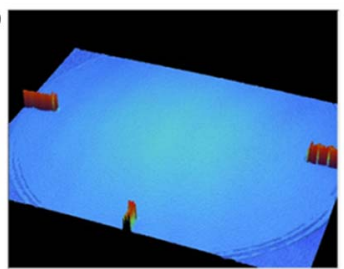

(c)
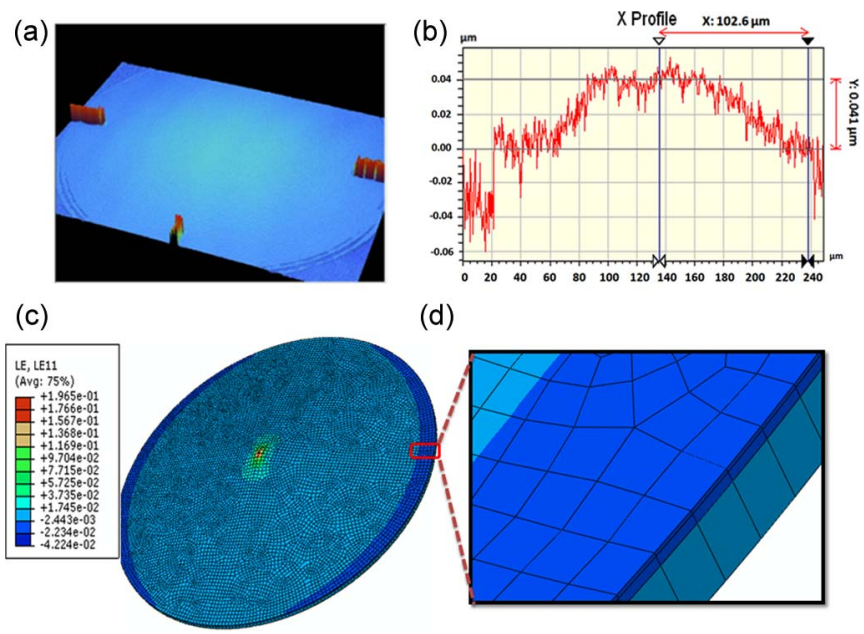

(d)

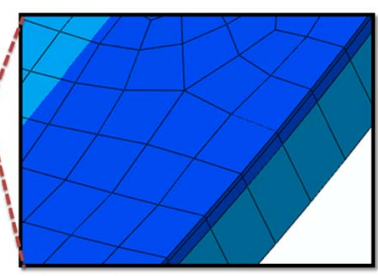

Fig. 2. (a) Three-dimensional topography of fabricated diaphragm structure (b) Curve showing surface roughness across the wafer. (c) ABAQUS simulation of the longitudinal strain in the SiNW. (d) Zoom-in picture showing the meshing and multilayered structure at the membrane edge of SiNW area.

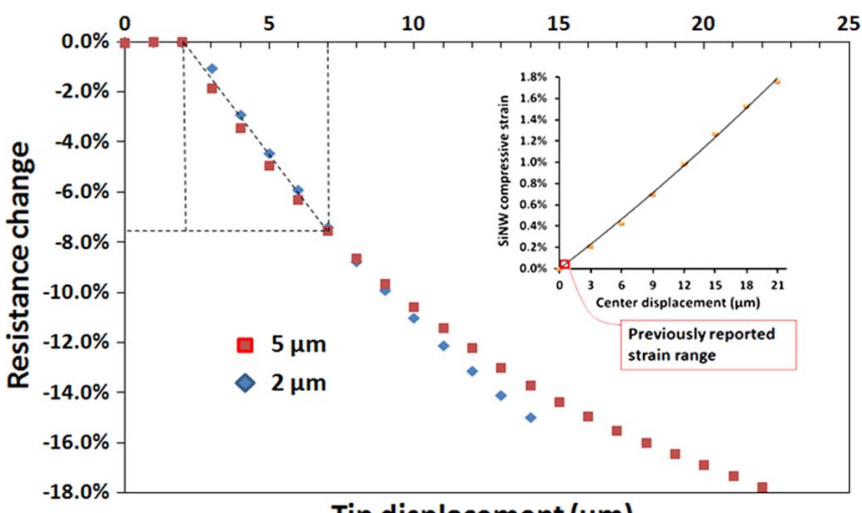

Tip displacement $(\mu \mathrm{m})$

Fig. 3. SiNW resistance change against tip displacement; inset shows the SiNW strain against the center displacement.

To experimentally characterize the microfabricated SiNW embedded in the MEMS devices, a specially fabricated tungsten needle is attached to a manipulator controlled by a PZT system using E-517 Digital Piezo Controller by Physik Instrumente. The diameter is $5 \mu \mathrm{m}$ with the fabricated needle tip which is around $300 \mathrm{~nm}$, which can be reasonably viewed as point in comparison with the $200-\mu \mathrm{m}$ diaphragm in diameter. The needle is deployed to push the diaphragm center, as illustrated by the white arrow mark in Fig. 1(a), thus deforming the membrane and transmitting the strain to the SiNW. Meanwhile, the electrical measurement of the SiNW resistance is conducted using the semiconductor parameter analyzer system (Agilent 4156C). The experiment is conducted on a probe station platform under a microscope. The tip is carefully aligned to be positioned directly on top of the center of the membrane and then moves in perpendicular to the membrane with $1-\mu \mathrm{m}$ displacement in each step. This approach takes advantage of the high fracture stress of silicon nitride and the PZT-based precise displacement control. Such a setup can exert quite high strain to the SiNW without demanding high pressure which is necessary to be applied in the bulge test [10]. Fig. 3 shows the measured resistance change against tip displacement for the sensors with 
2- $\mu \mathrm{m}$ and 5- $\mu \mathrm{m}$ SiNWs. The initial resistances derived from $I-V$ curves of linear characteristics for the $2-\mu \mathrm{m}$ and $5-\mu \mathrm{m}$ SiNWs are $88 \mathrm{k} \Omega$ and $226 \mathrm{k} \Omega$, respectively. It shows that the SiNW resistance keeps constant initially until the onset point at about $2.0 \mu \mathrm{m}$, indicating that the strain introduced by the tip is transmitted onto the SiNW. The resistance keeps dropping down to $17.7 \%$ for the $5-\mu \mathrm{m}$ SiNW and to $15.8 \%$ for the $2-\mu \mathrm{m} \mathrm{SiNW}$, respectively, as more displacement is continuously applied to the tip, while the membrane breaks when tip displacement reaches at $22 \mu \mathrm{m}$ and $14 \mu \mathrm{m}$, i.e., diaphragm center displacements of $20 \mu \mathrm{m}$ and $12 \mu \mathrm{m}$ accordingly. These data also exhibit a larger resistance change in comparison with previous reported data for bulky silicon under a compressive state, where such data are usually less than $8 \%$. The enlarged resistance range further proves the effectiveness of this testing approach. Moreover, the two curves show good linear behavior up to center displacement of about $5 \mu \mathrm{m}$ in Fig. 3. The 2- $\mu \mathrm{m} \mathrm{SiNW}$ curve gives slightly deeper slope than the one of $5-\mu \mathrm{m} \mathrm{SiNW}$ as indicated by the linear fitting line. This is due to the fact that the 5- $\mu \mathrm{m} \mathrm{SiNW} \mathrm{sensor} \mathrm{senses} \mathrm{wider} \mathrm{span} \mathrm{of} \mathrm{longitudinal} \mathrm{strain}$ across its length than the one of $2-\mu \mathrm{m} \mathrm{SiNW}$; in other words, the $5-\mu \mathrm{m}$ SiNW has lower average compressive strain than the $2-\mu \mathrm{m}$ SiNW under the same diaphragm center displacement.

There is nonlinear region observed for both cases when the center displacement is larger than $5 \mu \mathrm{m}$ until the fracture point. The resistance change decreases as the strain increases in this region, indicating that the gauge factor of the SiNW drops as the strain increases. Such nonlinear behavior has been investigated first by Suzuki et al. [13], and stress decoupling of the degenerate valence band into two bands of prolate and oblate ellipsoidal energy surface is proposed to explore the origin of the piezoresistance of p-silicon diffused layers. Our result is a further evidence to show the nonlinear behavior of the piezoresistance of $\mathrm{p}$-type silicon in an extended compressive range. Moreover, the $\mathrm{SiNW}$ is reported with giant factor by $\mathrm{He}$ and Yang [3]. The compressive range with appeared giant factor is lower than $0.06 \%$, which corresponds to the red box shown in the inset of Fig. 3. The evolution of the SiNW behavior under extended region of compressive strain could be of great value toward revealing the origin of the giant piezoresistive effect. From scientific point of view, the p-type of SiNW is not fully understood, and it requires more experimental effort [14], [15]. Our results revealed the SiNW properties in the large compressive strain region where it has not been reported until now. Additionally, in the practical applications, this result indicates that calibration is required to offset the nonlinearity of such sensors using the SiNW under large compressive strain.

\section{CONCLUSION}

In this letter, we have reported the experimental data of $\langle 110\rangle$ direction SiNW under extended compressive strain as large as $1.7 \%$ against $17.7 \%$ resistance change, while the nonlinear behavior is observed in the large compressive strain region. We successfully achieved measurement in large compressive strain, i.e., $1.7 \%$, by leveraging the intrinsic tensile stress of silicon nitride to produce a flat multilayered diaphragm consisting of silicon nitride, silicon oxide, and embedded SiNWs and by forming a strong membrane attributed to the high fracture stress of silicon nitride. Our results revealed the SiNW properties in the large compressive strain region where it has not been reported until now. It fills the missing link between actual behavior of SiNWs in sensor configuration and preliminary data of suspended SiNWs measured at bulky testing platform.

\section{ACKNOWLEDGMENT}

The author L. Lou would like to thank the National University of Singapore for Ph.D. research scholarship.

\section{REFERENCES}

[1] T. Toriyama, Y. Tanimoto, and S. Sugiyama, "Single crystal silicon nanowire piezoresistors for mechanical sensors," J. Microelectromech. Syst., vol. 11, no. 5, pp. 605-611, Oct. 2002.

[2] P. R. Nair and M. A. Alam, "Design considerations of silicon nanowire biosensors," IEEE Trans. Electron Devices, vol. 54, no. 12, pp. 34003408, Dec. 2007.

[3] R. He and P. Yang, "Giant piezoresistance effect in silicon nanowires," Nat. Nanotechnol., vol. 1, no. 1, pp. 42-46, Oct. 2006.

[4] P. Neuzil, C. C. Wong, and J. Reboud, "Electrically controlled giant piezoresistance in silicon nanowires," Nano Lett., vol. 10, no. 4, pp. 12481252, Apr. 2010.

[5] K. Reck, J. Richter, O. Hansen, and E. V. Thomsen, "Piezoresistive effect in top-down fabricated silicon nanowires," in Proc. IEEE MEMS, 2008, pp. $717-720$.

[6] B. Soon, P. Neuzil, C. Wong, J. Reboud, H. Feng, and C. Lee, "Ultrasensitive nanowire pressure sensor makes its debut," Procedia Eng., vol. 5, pp. 1127-1130, 2010.

[7] E. Lund and T. G. Finstad, "Design and construction of a four-point bending based set-up for measurement of piezoresistance in semiconductors," Rev. Sci. Instrum., vol. 75, no. 11, pp. 4960-4966, Nov. 2004.

[8] A. Lugstein, M. Steinmair, A. Steiger, H. Kosina, and E. Bertagnolli, "Anomalous piezoresistance effect in ultrastrained silicon nanowires," Nano Lett., vol. 10, no. 8, pp. 3204-3208, Aug. 2010.

[9] J. M. Chen and N. C. MacDonald, "Measuring the nonlinearity of silicon piezoresistance by tensile loading of a submicron diameter fiber using a microinstrument," Rev. Sci. Instrum., vol. 75, no. 1, pp. 276-278, Jan. 2004

[10] Y. Zhang, X. Y. Liu, C. H. Ru, Y. L. Zhang, L. X. Dong, and Y. Sun, "Piezoresistivity characterization of synthetic silicon nanowires using a MEMS device," J. Microelectromech. Syst., vol. 20, no. 4, pp. 959-967, Aug. 2011.

[11] S. Kozlovskiy and N. Sharan, "Piezoresistive effect in p-type silicon classical nanowires at high uniaxial strains," J. Comput. Electron., vol. 10, no. $1 / 2$, pp. $258-267$, Jun. 2011.

[12] V. Hatty, H. Kahn, and A. H. Heuer, "Fracture toughness, fracture strength, and stress corrosion cracking of silicon dioxide thin films," $J$. Microelectromech. Syst., vol. 17, no. 4, pp. 943-947, Aug. 2008.

[13] K. Suzuki, H. Hasegawa, and Y. Kanda, "Origin of the linear and nonlinear piezoresistance effects in p-type silicon," Jpn. J. Appl. Phys., vol. 23, no. 11, pp. L871-L874, Nov. 1984.

[14] K. Matsuda, "Strain-dependent hole masses and piezoresistive properties of silicon," in Proc. 10th IWCE, 2004, pp. 173-174.

[15] A. A. Barlian, W.-T. Park, J. R. Mallon, Jr., A. J. Rastegar, and B. L. Pruitt, "Review: Semiconductor piezoresistance for microsystems," Proc. IEEE, vol. 97, no. 3, pp. 513-552, Mar. 2009. 